\title{
A note on the use of analytical and domain discretisation methods for the analysis of some phenomena in engineering physics
}

\author{
Dragan Poljak(1), Silvestar Sesnić(1), Ante Rubić(1), Enora Maze(2) \\ (1) University of Split, Split, CROATIA \\ e-mail: dragan.poljak@fesb.hr \\ (2) Blaise Pascal University, Clermont-Ferrand, FRANCE
}

\section{SUMMARY}

The paper reviews some applications of the well-established analytical and domain discretisation methods (finite difference method-FDM, finite element method-FEM) in the modelling of the magnetohydrodynamics (MHD) phenomena for the fusion-related research and quantum structures for use in nanodevices. Hence, Grad-Shafranov Equation (GSE) for the plasma equilibrium has been implemented for certain simple excitation forms, and the results obtained for the rectangular plasma have been presented. Furthermore, the stationary Schrödinger equation is solved analytically and numerically via FDM and FEM, respectively.

KEY WORDS: finite difference method; finite element method; Grad-Shafranov equation; magnetohydrodynamics; nanodevices; plasma shape; Schrödinger equation.

\section{INTRODUCTION}

The shape of the real-world geometries in most of the realistic scenarios in physics and engineering practice are usually highly irregular. There are also difficulties due to nonhomogeneities, anisotropy, non-linearity, prescribing appropriate boundary conditions, etc. A way to overcome at least some of the aforementioned difficulties is to implement certain numerical modelling techniques. One of the most general classifications of numerical methods is to domain discretisation methods (based on the solution of a certain partial differential equations (PDE)) and boundary discretisation methods (based on the solution of a given integral equation) [1]. A trade-off between the use of domain and boundary methods could be found elsewhere, e.g. in [1], [2].

On the other hand, a starting point in studying physical phenomena is to find the analytical solution for problems involving regular geometries and homogeneous domains. 
The present paper reviews some analytical approaches, and, in particular, some aspects of the use of domain methods (FDM and FEM) in the modelling of some phenomena in magnetohydrodynamics (MHD) and quantum mechanics. The paper deals with the solution of Grad-Shafranov equation (GSE) arising from the plasma physics and the Schrödinger equation stemming from the analysis of nanodevices.

The principal advantage of domain methods is that the Green function is not used in the formulation. The domain methods always result in sparse matrices thus appreciably reducing the computational cost. The main drawback of such methods is that they are not suitable for the treatment of unbounded domains.

The use of MHD models is the simplest theory for the description of ionized gasses, i.e. the simplest theoretical approach for the analysis of the electrically conducting fluids called plasma. Even though the MHD approach is far from the rigorous description of any realistic plasma configuration of interest, it still provides the theoretical basis for understanding the global configuration of magnetised plasma. As plasma represents a good conductor at high temperatures, plasma shaping and confinement can be carried out by high-intensity magnetic fields. Particular configurations of plasma are governed by the Grad-Shafranov equation (GSE) whose general form cannot be solved analytically [3]-[5]. In the last few decades, there have been number of papers dealing with the numerical treatment of tokamak plasma, e.g. [6]-[7].

The numerical modelling of the main plasma region is a less difficult task compared to the modelling of plasma edge in tokamak [7]. In fact, the problem of prescribing appropriate boundary conditions to be imposed at the plasma edge has still not been solved satisfactorily in the physical sense. The reasons are manifold and have been discussed elsewhere, e.g. in [7].

This paper reviews the use of the standard FDM and FEM procedures featuring isoparametric elements for the solution of rectangular plasma assuming the source term (provided in the form of current density) to be monomial [5].

The obtained results are in a good agreement with the numerical results published in [3].

Furthermore, many phenomena in quantum transport, condensed matter physics, optics, nanodevices, etc. are governed by the Schrödinger equation [8]-[11]. The main difficulty in the realistic scenarios is to prescribe appropriate boundary conditions, as the Schrödinger equation itself is posed in an unbounded domain.

The modelling of semiconductor structures is of particular interest in both physics and engineering. In some applications, such as the analysis of nanowires, a combined classical/quantum physics approach is used featuring the hybrid Poisson/Schrödinger equation approach, [8]. In fact, as the size of nanowires approaches to nano scales, the quantum effects become crucial to understand and design such structures. Hence, the charge or scalar potential distribution could be determined by solving the Poisson/Schrödinger equation.

Finally, it should be emphasised that the present paper is a direct extension of the conference paper [12], whereas some parts of this review paper are taken from the conference papers [13] and [14].

\section{MODELLING OF GRAD-SHAFRANOV EQUATION}

The behaviour of dynamics phenomena in tokamaks can be analysed by solving the combined equations of electromagnetics and fluid dynamics. Electromagnetic modelling of the dynamics 
phenomena in tokamaks is governed by the quasi-stationary Maxwell equations. MHD equilibrium in an axisymmetric plasma shape, as depicted in Figure 1, is considered.

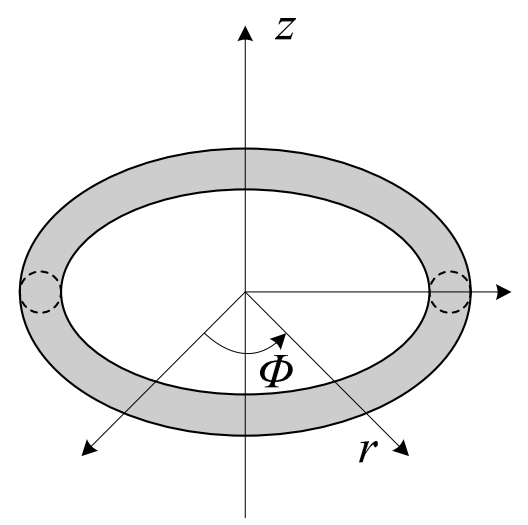

Fig. 1 Toroidal geometry

The force balance equation is given by Eq. [3]:

$$
\vec{J} \times \vec{B}=\nabla p
$$

where $J$ is the current density, $B$ is magnetic flux density and $p$ stands for the kinetic pressure. In cylindrical coordinates $(r, \phi, z)(1)$ is expressed, as follows:

$$
\vec{J}_{p o l} \times B_{\varphi} \vec{e}_{\varphi}+J_{\varphi} \vec{e}_{\varphi} \times \vec{B}_{p o l}=\nabla p
$$

where $\vec{J}_{p o l}$ is the poloidal current density, $\vec{B}_{p o l}$ is the poloidal magnetic flux density and $\vec{e}_{\varphi}$ is the unit vector in toroidal direction.

Now, the poloidal quantities can be written:

$$
\begin{gathered}
\vec{B}_{p o l}=\frac{1}{r}\left(\nabla \Psi \times \vec{e}_{\varphi}\right) \\
\vec{J}_{p o l}=\frac{1}{r}\left(\nabla f \times \vec{e}_{\varphi}\right)
\end{gathered}
$$

where $\psi$ is the poloidal magnetic flux, while $f$ is defined as:

$$
f=\frac{r B_{\varphi}}{\mu_{0}}
$$

Now, taking into account:

$$
\vec{e}_{\varphi} \cdot \nabla \Psi=\vec{e}_{\varphi} \cdot \nabla f=0
$$

relation (2) becomes:

$$
-\frac{1}{r} B_{\varphi} \nabla f+\frac{1}{r} J_{\varphi} \nabla \psi=\nabla p
$$

By utilising relations:

$$
\begin{aligned}
& \nabla p(\Psi)=\frac{\partial p}{\partial \Psi} \nabla \Psi \\
& \nabla f(\Psi)=\frac{\partial f}{\partial \Psi} \nabla \Psi
\end{aligned}
$$


expression (7) can be written:

$$
J_{\varphi}=r \frac{\partial p}{\partial \psi}+B_{\varphi} \frac{\partial f}{\partial \psi}=r \frac{\partial p}{\partial \psi}+\mu_{0} \frac{1}{r} f \frac{\partial f}{\partial \psi}
$$

As the magnetic flux density and current density are related by Ampere law:

$$
\nabla \times \vec{B}=\mu_{0} \vec{J}
$$

by taking into account (3) one obtains:

$$
-\mu_{0} r J_{\varphi}=r \frac{\partial}{\partial r} \frac{1}{r} \frac{\partial \psi}{\partial r}+\frac{\partial^{2} \psi}{\partial z^{2}}
$$

Finally, by combining (10) and (12) yields:

$$
r \frac{\partial}{\partial r} \frac{1}{r} \frac{\partial \psi}{\partial r}+\frac{\partial^{2} \psi}{\partial z^{2}}=-\mu_{0} r^{2} \frac{\partial p}{\partial \psi}-\mu_{0}^{2} f \frac{\partial f}{\partial \psi}
$$

which is one of the commonly used form of the Grad-Shafranov equation (GSE).

An alternative useful form of GSE is given by using elliptic operator:

$$
\left[r \frac{\partial}{\partial r}\left(\frac{1}{r} \frac{\partial \psi}{\partial r}\right)+\frac{\partial^{2} \psi}{\partial z^{2}}\right]=-\mu_{0} r J_{\varphi}
$$

where $J$ is the toroidal component of the plasma current.

Equation (14) can be solved analytically only for a few special cases, however for additional problems with a higher degree of complexity, the numerical solution is necessary.

\subsection{ANALYTICAL SOLUTION}

Various analytical solutions to the GSE have been derived by researchers through the recent years [15]. As it has been stated, the analytical solutions are essential in describing various parameters that are involved in real tokamak scenarios as they are well suited for benchmarking various numerical codes. In this section, four different analytical solutions will be outlined, as well as the emphasis to their applications.

Starting from a slightly modified version of (13), i.e. from equation:

$$
\frac{\partial^{2} \psi}{\partial r^{2}}-\frac{1}{r} \frac{\partial \psi}{\partial r}+\frac{\partial^{2} \psi}{\partial z^{2}}=-\mu_{0}^{2} f \frac{d f}{d \psi}-\mu_{0} r^{2} \frac{d P}{d \psi}
$$

in order to obtain any solution corresponding to the realistic source functions that appear on the right-hand side of the GSE, it is necessary to determine possible solutions of the homogeneous equation:

$$
\frac{\partial^{2} \psi}{\partial r^{2}}-\frac{1}{r} \frac{\partial \psi}{\partial r}+\frac{\partial^{2} \psi}{\partial z^{2}}=0
$$

Solution of (16) can be obtained by the separation of variables and is given by:

$$
\psi_{0}(r, z)=\left(c_{1} r J_{1}(k r)+c_{2} r Y_{1}(k r)\right)\left(c_{3} e^{k z}+c_{4} e^{-k z}\right)
$$

On the other hand, the solutions can also be based on the series expansion [16]: 


$$
\psi_{0}=\sum_{n=0,2, \ldots} f_{n}(r) z^{n}
$$

provided that each expansion term satisfies the following Eq. [16]:

$$
r \frac{d}{d r}\left(\frac{1}{r} \frac{d f_{n}(r)}{d r}\right)=-(n+1)(n+2) f_{n+2}, n=0,2, \ldots
$$

One of the possible solutions satisfying these conditions which is suitable for further implementation is given by [16]:

$$
\psi_{0}(r, z)=c_{1}+c_{2} r^{2}+c_{3}\left(r^{4}-4 r^{2} z^{2}\right)+c_{4}\left(r^{2} \ln r-z^{2}\right)
$$

The Solov'ev equilibrium is the simplest solution of the inhomogeneous GSE [15], being widely used in studies of plasma equilibrium studies, transport and MHD stability analysis, respectively.

The source functions in Solov'ev equilibrium are linear in $\psi$ and are given by [17]:

$$
P(\psi)=\frac{A}{\mu_{0}} \psi, f^{2}(\psi)=2 B \psi+F_{0}^{2}
$$

The use of source functions (20) yields the following variant of the GSE:

$$
\frac{\partial^{2} \psi}{\partial r^{2}}-\frac{1}{r} \frac{\partial \psi}{\partial r}+\frac{\partial^{2} \psi}{\partial z^{2}}=A r^{2}+B
$$

with the corresponding solution:

$$
\psi(r, z)=\psi_{0}(r, z)-\frac{A}{8} r^{4}-\frac{B}{2} z^{2}
$$

It is worth noting that a number of plasma shapes can be generated by using (23). However, the current profile of this solution is restricted, as two free parameters $A$ and $B$ allow only the selection of the plasma current and the ratio of the volume-averaged particle pressure to the average poloidal magnetic field pressure along the plasma boundary.

The Herrnegger-Maschke solutions of the GSE for a parabolic source functions were originally reported in [15] and can be written as follows:

$$
P(\psi)=\frac{C}{2 \mu_{0}} \psi^{2}, f^{2}(\psi)=D \psi^{2}+F_{0}^{2}
$$

In this case, the GSE simplifies into:

$$
\frac{\partial^{2} \psi}{\partial r^{2}}-\frac{1}{r} \frac{\partial \psi}{\partial r}+\frac{\partial^{2} \psi}{\partial z^{2}}=C r^{2} \psi+D \psi
$$

The solution of (25) can be given in the form of Coulomb wave functions:

$$
\psi=\alpha\left(F_{0}(\eta, x)+\gamma G_{0}(\eta, x)\right) \cos (k z)
$$

As in the case of the Solov'ev equilibrium, the Herrnegger-Maschke solutions have only two free parameters ( $C$ and $D$ ), which enables one to independently specify the plasma current and the pressure ratio, respectively.

Innovative source functions have been introduced by Mc Carthy [15]. These source functions involve a linear dependence of pressure and a quadratic dependence of the current profile: 


$$
P(\psi)=\frac{S}{\mu_{0}} \psi, f^{2}(\psi)=T \psi^{2}+2 U \psi+F_{0}^{2}
$$

Inserting (27) in (15) yields:

$$
\frac{\partial^{2} \psi}{\partial r^{2}}-\frac{1}{r} \frac{\partial \psi}{\partial r}+\frac{\partial^{2} \psi}{\partial z^{2}}=-S r^{2}-T \psi-U
$$

Note that the term $S r^{2}+U$ satisfies the equation $\Delta^{*}\left(S r^{2}+U\right)=0$, hence the following homogeneous equation has to be solved:

$$
\frac{\partial^{2} \psi_{h}}{\partial r^{2}}-\frac{1}{r} \frac{\partial \psi_{h}}{\partial r}+\frac{\partial^{2} \psi_{h}}{\partial z^{2}}+T \psi_{h}=0
$$

Once $\psi_{h}$ is found, the solution can be written in the form:

$$
\psi=\psi_{h}-\frac{S}{T} r^{2}-\frac{U}{T}
$$

and the following differential equation is obtained:

$$
\frac{\partial^{2} \psi}{\partial r^{2}}-\frac{1}{r} \frac{\partial \psi}{\partial r}+\frac{\partial^{2} \psi}{\partial z^{2}}=-T \psi-S r^{2}-U
$$

Equation (31) can be solved by the separation of variables which yields the following two ordinary differential equations:

$$
\begin{gathered}
\frac{\partial^{2} H(z)}{\partial z^{2}}+k^{2} H(z)=0 \\
\frac{\partial^{2} G(r)}{\partial r^{2}}-\frac{1}{r} \frac{\partial G(r)}{\partial r}-\left(k^{2}-T\right) G(r)=0
\end{gathered}
$$

The corresponding solutions of (32) and (33) are given by:

$$
\begin{gathered}
H(z)=c_{1} e^{j k z}+c_{2} e^{-j k z} \\
G(r)=r B_{1}(a r)
\end{gathered}
$$

where $B_{1}$ represents the family of Bessel functions and parameter $a$ satisfies the Eq. [15]:

$$
a^{2}= \pm\left(T-k^{2}\right)
$$

More details can be found in [15].

This family of solutions has a current profile with 3 independent parameters thus providing one to independently specify the plasma current density, pressure ratio and one shape moment such as the internal inductance [15]. Consequently, it is possible to fit experimental configurations in a manner consistent with the external magnetic measurements.

In order to obtain the exact solution out of Eqs. (34) and (35) for various real scenarios, the numerical solution of the free boundary problem (with a conventional equilibrium solver) and the subsequent projection of the numerically calculated solution onto the exact solutions via the least squares fitting procedure is implemented [15]. The obtained solution can be described as: 


$$
\begin{aligned}
& \psi=c_{1}+c_{2} r^{2}+r J_{1}(p r)\left(c_{3}+c_{4} z\right)+c_{5} \cos p z+c_{6} \sin p z+ \\
& +r^{2}\left(c_{7} \cos p z+c_{8} \sin p z\right)+c_{9} \cos p \sqrt{r^{2}+z^{2}}+ \\
& +c_{10} \sin p \sqrt{r^{2}+z^{2}}+r J_{1}(v r)\left(c_{11} \cos q z+c_{12} \sin q z\right)+ \\
& +r J_{1}(q r)\left(c_{13} \cos v z+c_{14} \sin v z\right)+ \\
& +r Y_{1}(v r)\left(c_{15} \cos q z+c_{16} \sin q z\right)+ \\
& +r Y_{1}(q r)\left(c_{17} \cos v z+c_{18} \operatorname{sinvz}\right)+
\end{aligned}
$$

where the vector of coefficients $c_{i}$ can be found in [15].

An example of a predictive reversed shear equilibrium using ASDEX upgrade field coils and vessel can be described in a similar fashion with the Eq. [15]:

$$
\begin{aligned}
& \psi=c_{1}+c_{2} r^{2}+r I_{1}(p r)\left(c_{3}+c_{4} z\right)+c_{5} \cosh p z+c_{6} \sinh p z+ \\
& +r^{2}\left(c_{7} \cosh p z+c_{8} \sinh p z\right)+c_{9} \cosh p \sqrt{r^{2}+z^{2}}+ \\
& +c_{10} \sinh p \sqrt{r^{2}+z^{2}}+r I_{1}(v r)\left(c_{11} \cosh q z+c_{12} \sinh q z\right)+ \\
& +r I_{1}(q r)\left(c_{13} \cosh v z+c_{14} \sinh v z\right)+ \\
& +r K_{1}(v r)\left(c_{15} \cosh q z+c_{16} \sinh q z\right)+ \\
& +r K_{1}(q r)\left(c_{17} \cosh v z+c_{18} \sinh v z\right)+
\end{aligned}
$$

More mathematical details are available in [15].

\subsection{COMPUTATIONAL EXAMPLES}

The results presented in this subsection correspond to the results for the tokamak equilibrium obtained by using analytical solutions (37) and (38) derived by Mc Charty. In Figure 2, the results for poloidal flux contours for ASDEX Upgrade discharge \# 10 966, $t=1.242 \mathrm{~s}$, calculated by using (37), are presented.

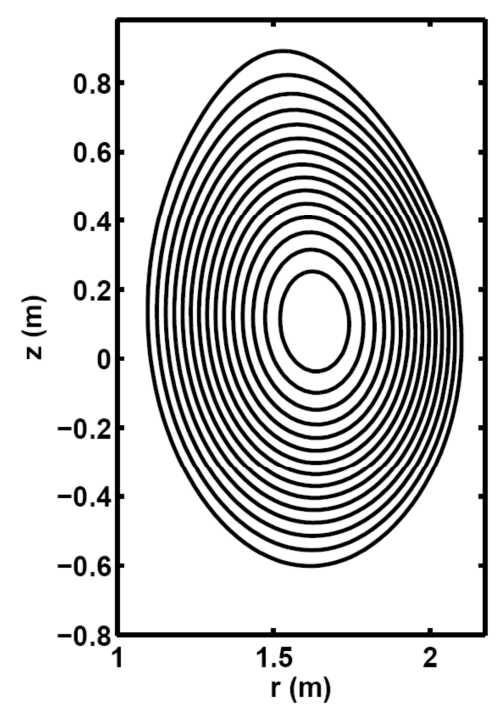

Fig. 2 Exact GSE solution for ASDEX Upgrade discharge \# $10966, t=1.24 \mathrm{~s}$ 
The highest value for the poloidal magnetic flux $\psi_{\max }=1.3 \mathrm{Tm}^{2}$ is observed at the centre of the tokamak plasma, as it is expected, whereas the final contour (called separatrix) defines the area where the value of the magnetic flux is equal to zero.

For the solution depicted in Figure 3, the similar behaviour of the plasma flux can be observed, with a somewhat different shape of plasma which is governed by the specifics of the defined discharge pulse.

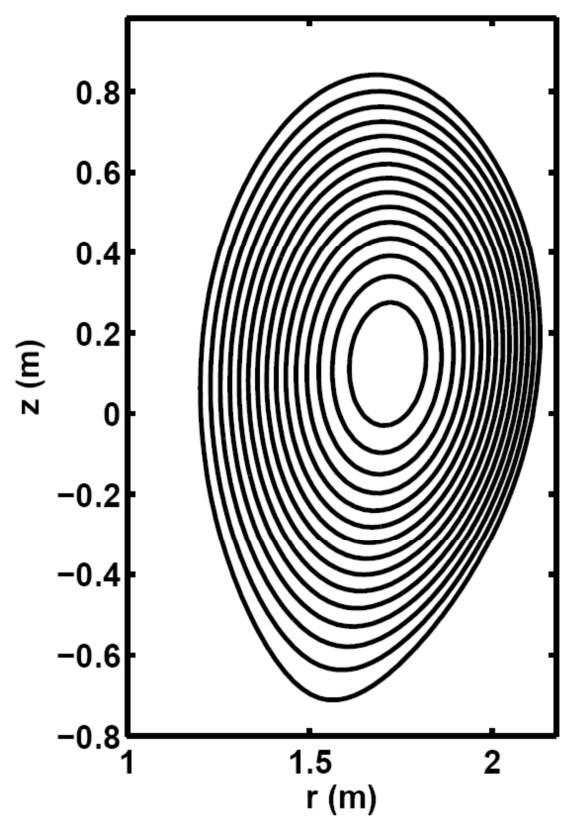

Fig. 3 Exact GSE solution for ASDEX Upgrade discharge \# $10958, t=5.20 \mathrm{~s}$

The maximum value of the magnetic flux is somewhat higher $\psi_{\max }=1.4 \mathrm{Tm}^{2}$.

An example of the predictive reversed shear equilibrium by using ASDEX upgrade field coils and vessel geometry is shown in Figure 4 and is calculated by using (38).

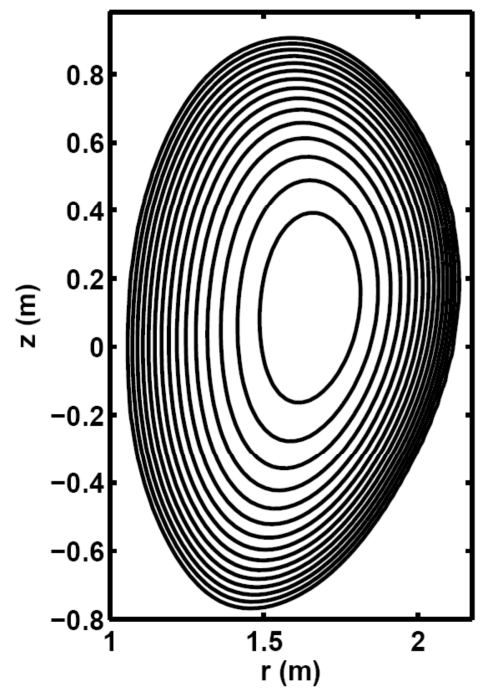

Fig. 4 Exact GSE solution for a predictive reversed ASDEX Upgrade equilibrium 
The value of the maximum flux is significantly lower than in the previous examples and is given as $\psi_{\max }=0.6 \mathrm{Tm}^{2}$.

\subsection{SOLUTION BY FINITE DIFFERENCE METHOD (FDM)}

For the application of FDM to solve (13), GSE could be written in the following form:

$$
-\left(\frac{\partial^{2} \Psi}{\partial r^{2}}+\frac{\partial^{2} \Psi}{\partial z^{2}}-\frac{1}{r} \frac{\partial \Psi}{\partial r}\right)=\mu_{0} r J_{\varphi}
$$

Now the FDM discretisation yields:

$$
\begin{aligned}
& \frac{\psi(r+\Delta r, z)-2 \psi(r, z)+\psi(r-\Delta r, z)}{\Delta r^{2}}+ \\
& +\frac{\psi(r, z+\Delta z)-2 \psi(r, z)+\psi(r, z-\Delta z)}{\Delta z^{2}}+ \\
& +\frac{1}{r} \frac{\psi(r+\Delta r, z)-\psi(r-\Delta r, z)}{2 \Delta r}=-\mu_{0} r J_{\varphi}
\end{aligned}
$$

Finite difference Eq. (40) is applied to each node and can be solved by prescribing certain boundary conditions [5].

\subsection{SOLUTION BY FINITE ELEMENT METHOD (FEM)}

In order to implement the FEM solution, GSE form (39) is also used. Taking the scalar product over the calculation domain yields:

$$
-\int_{\Omega}\left(\frac{\partial^{2} \Psi}{\partial r^{2}}+\frac{\partial^{2} \Psi}{\partial z^{2}}-\frac{1}{r} \frac{\partial \Psi}{\partial r}\right) W_{j} d \Omega=\int_{\Omega} \mu_{0} r J_{\varphi} W_{j} d \Omega
$$

and the performance of some mathematical manipulations leads to the weak formulation of GSE:

$$
-\int_{\Gamma} \frac{\partial \Psi}{\partial n} W_{j} d \Gamma+\int_{\Omega}\left(\frac{\partial \Psi}{\partial r} \frac{\partial W_{j}}{\partial r}+\frac{\partial \Psi}{\partial z} \frac{\partial W_{j}}{\partial z}\right) d \Omega+\int_{\Omega} \frac{1}{r} \frac{\partial \Psi}{\partial r} W_{j} d \Omega=\int_{\Omega} \mu_{0} r J_{\varphi} W_{j} d \Omega
$$

By using the triangular elements and linear shape functions, the solution over an element is given by:

$$
\psi(r, z)=\sum_{i=1}^{3} \alpha_{i} f_{i}(r, z)
$$

Now, by choosing the same shape and test functions (Galerkin-Bubnov scheme):

$$
W_{j}(r, z)=f_{j}(r, z)
$$

the local matrix system on the element is obtained:

$$
[A]\{\alpha\}=\{B\}
$$

where FEM matrix and excitation vector coefficients are: 
D. Poljak, S. Sesnić, A. Rubić, E. Maze: A note on the use of analytical and domain discretisation methods for the analysis of some phenomena in engineering physics

$$
\begin{gathered}
A_{j i}=\int_{\Omega_{e}}\left[\frac{\partial f_{j}(r, z)}{\partial r} \frac{\partial f_{i}(r, z)}{\partial r}+\frac{\partial f_{j}(r, z)}{\partial z} \frac{\partial f_{i}(r, z)}{\partial z}\right] d \Omega_{e}+\int_{\Omega_{e}} \frac{1}{r} f_{j}(r, z) \frac{\partial f_{i}(r, z)}{\partial r} d \Omega_{e} \\
B_{j i}=\mu_{0} \int_{x_{1}}^{x_{2}} r J_{\varphi} f_{j}(r, z) d \Omega_{e}
\end{gathered}
$$

Integral (46) is solved analytically, whereas the integral (47) can generally be calculated numerically by using the Gaussian quadrature formulas.

\subsection{COMPUTATIONAL EXAMPLES}

The computational examples are related to rectangular plasma. Figures 5 to 7 show the distribution of flux $\Psi(W b)$ for the rectangular plasma obtained by FDM and FEM $\mu_{0} J_{\varphi}=1$, $\mu_{0} r J_{\varphi}=r^{3} z^{2}$ and $\mu_{0} r J_{\varphi}=r^{2} z^{3}$, respectively.

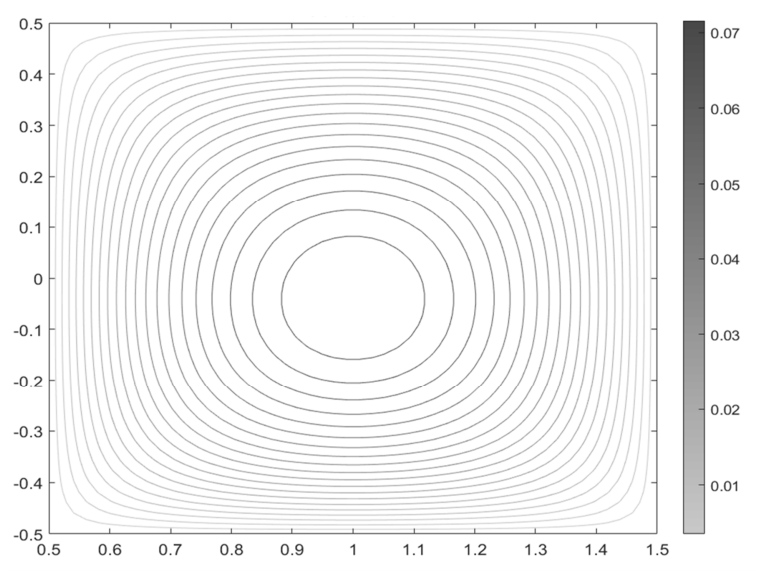

a) FDM solution

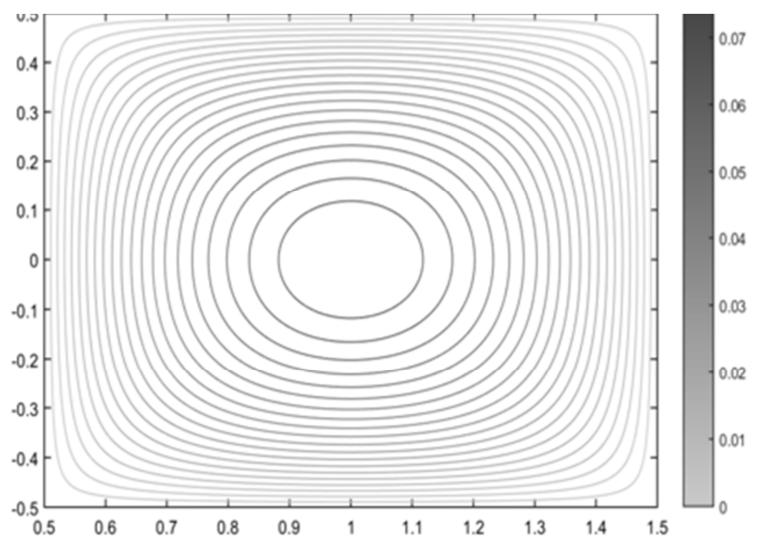

b) FEM solution

Fig. $5 \psi(W b)$ for rectangular plasma $\left.-\mu_{0} r\right\rfloor_{\varphi}=1$ 
D. Poljak, S. Sesnić, A. Rubić, E. Maze: A note on the use of analytical and domain discretisation methods for the analysis of some phenomena in engineering physics

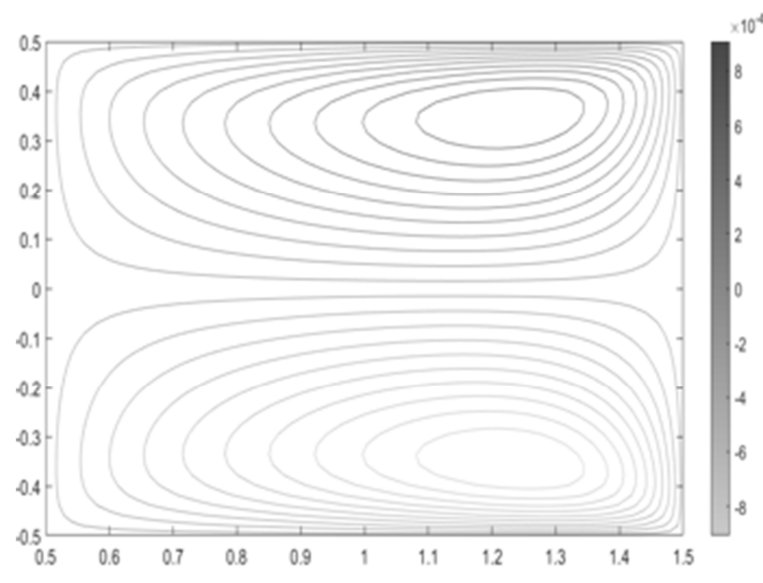

a) FDM solution

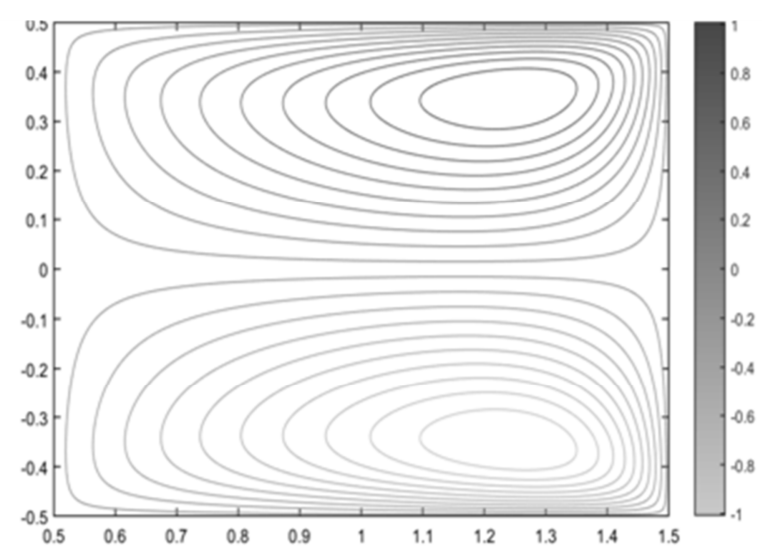

b) FEM solution

Fig. $6 \Psi(W b)$ for rectangular plasma $-\mu_{0} r J_{\varphi}=r^{2} z^{3}$ 
D. Poljak, S. Sesnić, A. Rubić, E. Maze: A note on the use of analytical and domain discretisation methods for the analysis of some phenomena in engineering physics

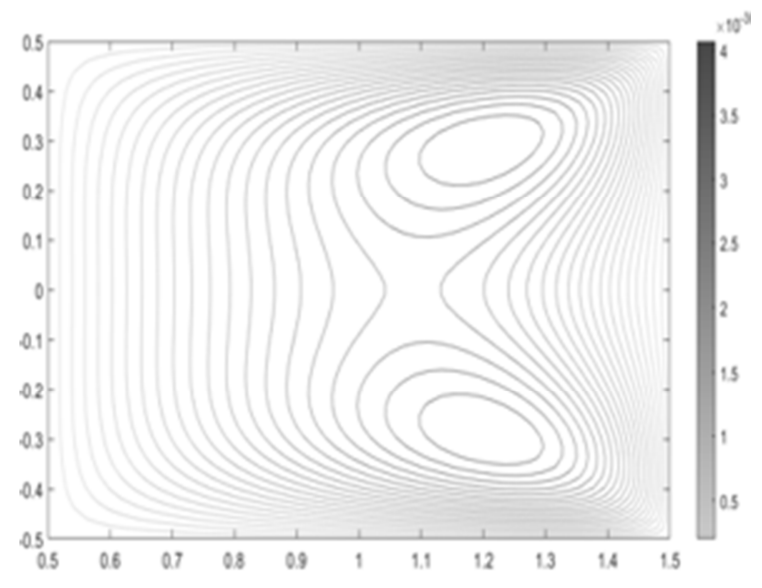

a) FDM solution

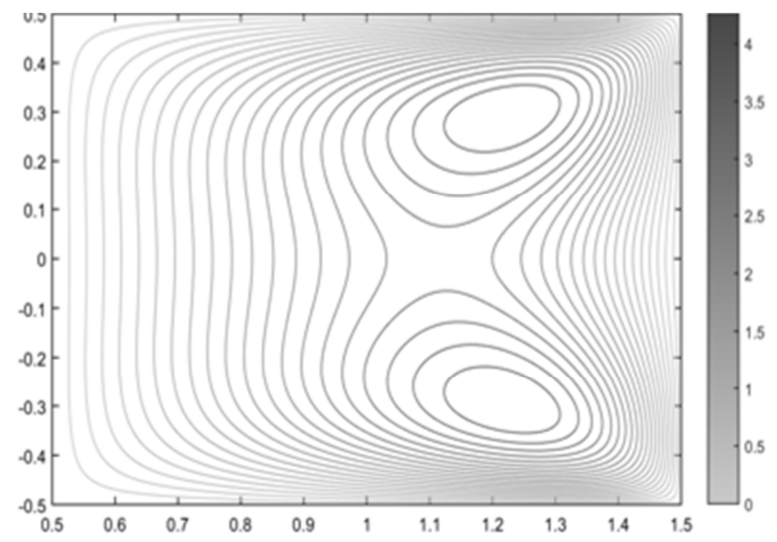

b) FEM solution

Fig. $7 \Psi(W b)$ for rectangular plasma $-\mu_{0} r J_{\varphi}=r^{3} z^{2}$

The numerical results obtained by FEM are in a satisfactory agreement with the results published in [5].

\section{MODELLING OF SCHRÖDINGER EQUATION}

The classical form of the time-independent Schrödinger equation can be written as follows [11]:

$$
H \psi=E \psi
$$

where $\Psi$ is the wave function, $E$ is the energy of the quantum particle of mass $m$ moving within the interval $(0, L)$ and $H$ is the Hamiltonian:

$$
H=-\frac{\hbar^{2}}{2 m} \frac{\partial^{2}}{\partial x^{2}}+V(x)
$$

where $V(x)$ is the corresponding potential function, while the reduced Planck constant is given by: 


$$
\hbar=\frac{h}{2 \pi}
$$

The energy spectrum of the quantum particle can be determined by solving the Schrödinger Eq. (48) provided that the boundary conditions for the wave function $\Psi$ are prescribed.

\subsection{ANALYTICAL SOLUTION}

Assuming the particle to be captured inside the potential well with $V=0$, and cannot be located outside the interval $(O, L)$ with the boundary conditions:

$$
\psi(0)=\psi(L)=0
$$

One-dimensional Schrödinger equation simplifies into:

$$
-\frac{\partial^{2} \psi}{\partial x^{2}}=k^{2} \psi
$$

The analytical solution of (50) is given by:

$$
\psi(x)=A \sin k x+B \cos k x
$$

Inserting the boundary conditions (49) yields:

$$
B=0
$$

and from:

$$
\sin k L=0
$$

The wave number $k$ is obtained:

$$
k=\frac{n \pi}{L}
$$

and the wave function is:

$$
\psi(x)=A \sin \left(\frac{n \pi}{L} k\right)
$$

Probability of the existence of the quantum particle within the well, i.e. within the observed interval $(0, L)$, is equal to one, i.e.

$$
\int_{0}^{L}|\psi(x)|^{2} d x=1
$$

Hence, inserting (55) into (56) one obtains:

$$
A^{2} \int_{0}^{L} \sin ^{2}\left(\frac{n \pi}{L} k\right) d x=1
$$

and it simply follows:

$$
A=\sqrt{\frac{2}{L}}
$$

Finally, the solution of the Schrödinger Eq. (48) is:

$$
\psi(x)=\sqrt{\frac{2}{L}} \sin \left(\frac{n \pi}{L} x\right)
$$


The Schrödinger equation for the case of the three-dimensional potential well can be handled by using a similar procedure.

\subsection{SOLUTION BY FINITE DIFFERENCE METHOD (FDM)}

The application of FDM to the one-dimensional Schrödinger equation:

$$
-\frac{\hbar}{2 m} \frac{\partial^{2} \psi(x)}{\partial x^{2}}+V(x) \psi=E \psi(x)
$$

for the case of the particle inside the potential well with $V=0$ yields:

$$
-\frac{\hbar}{2 m} \frac{\psi(x+\Delta x)-2 \psi(x)+\psi(x-\Delta x)}{\Delta x^{2}}+V(x) \psi(x)=E \psi(x)
$$

and results in the system of $N$ equations with $N$ unknowns.

\subsection{SOLUTION BY FINITE ELEMENT METHOD (FEM)}

By applying the weighted residual approach to the Schrödinger Eq. (60), one obtains:

$$
-\int_{0}^{L} \frac{\hbar}{2 m} \frac{\partial^{2} \psi}{\partial x^{2}} W_{j} d x+\int_{0}^{L} V(x) \psi W_{j} d x=\int_{0}^{L} E \psi W_{j} d x
$$

and by utilising the weak formulation, it follows:

$$
\int_{0}^{L} \frac{\partial \psi}{\partial x} \frac{\partial W_{j}}{\partial x} d x+\frac{2 m}{\hbar^{2}} \int_{0}^{L} V(x) \psi W_{j} d x=\frac{2 m}{\hbar^{2}} E \int_{0}^{L} \psi W_{j} d x+\left.\frac{2 m}{\hbar^{2}} \frac{\partial \psi}{\partial x} W_{j}\right|_{0} ^{L}
$$

The approximate solution is given in terms of the linear combination of coefficients $\alpha_{i}$ and shape functions $f_{i}$ :

$$
\psi(x)=\{f\}^{T}\{\alpha\}
$$

where the linear shape functions are given by:

$$
f_{1}(x)=\frac{x_{2}-x}{\Delta x}, f_{2}(x)=\frac{x-x_{1}}{\Delta x}, \Delta x=x_{2}-x_{1}
$$

By discretising the calculation domain and applying the Galerkin-Bubnov procedure $W_{j}=f_{j}$, the following matrix equation is obtained:

$$
[A]\{\alpha\}=[B]\{\alpha\}[E]
$$

where $[E]$ is a diagonal matrix representing the particle energy levels in different states.

FEM matrix and excitation vector coefficients are:

$$
\begin{gathered}
A_{j i}=\int_{x_{1}}^{x_{2}} \frac{\partial f_{j}(x)}{\partial x} \frac{\partial f_{i}(x)}{\partial x} d x+\frac{2 m}{\hbar^{2}} \int_{x_{1}}^{x_{2}} V(x) f_{j}(x) f_{i}(x) d x \\
B_{j i}=\frac{2 m}{\hbar^{2}} \int_{x_{1}}^{x_{2}} f_{j}(x) f_{i}(x) d x
\end{gathered}
$$


Expression (66) is eigenvalue equation. Each element of $[E]$ matrix is then an eigenvalue (certain energy level). Number of solutions corresponds to the number of quantised energy levels that particle can occupy inside an infinite potential well.

Figures 8 to 10 show the probability density function $|\psi|^{2}$ for different values of $n$ calculated via FDM and FEM.

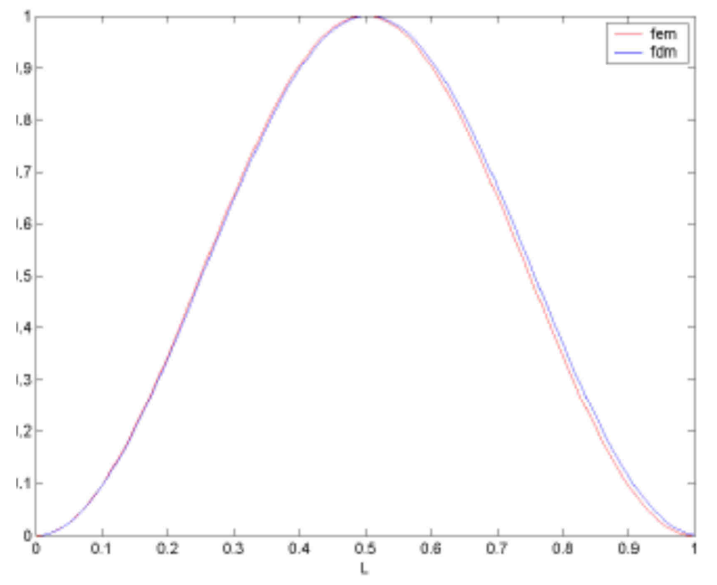

Fig. $8|\psi|^{2}$ Probability density function for $N=100$ and $n=1$

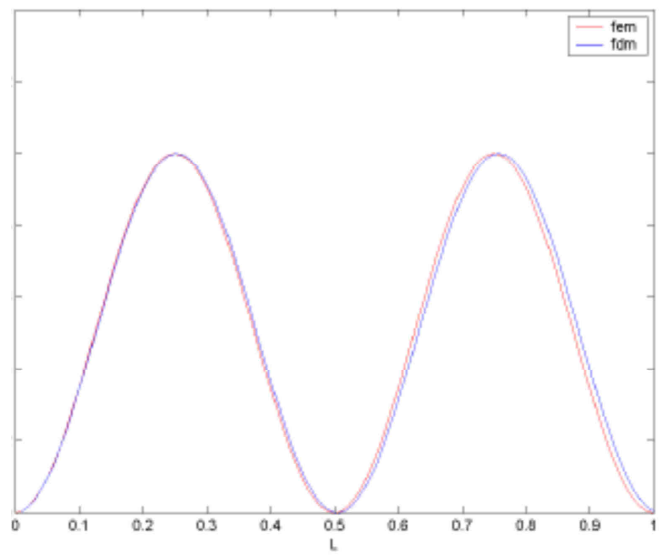

Fig. $9|\psi|^{2}$ Probability density function for $N=100$ and $n=2$ 


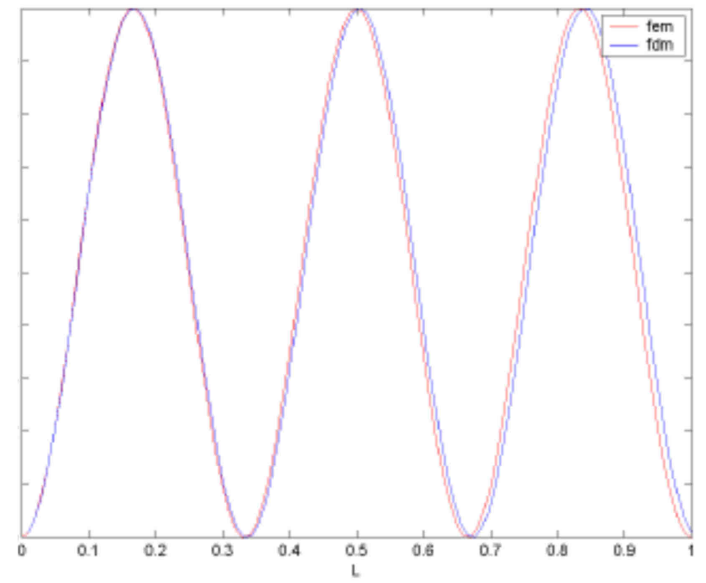

Fig. $10|\psi|^{2}$ Probability density function for $N=100$ and $n=3$

The numerical results obtained by FDM and FEM, respectively, agree satisfactorily. The analysis presented so far is a useful starting point for the analysis of practical scenarios whose solution cannot be obtained in the close form.

\section{CONCLUDING REMARKS}

The paper reviews the use of the standard analytical and domain discretisation methods (FDM and FEM), respectively, for the treatment of MHD phenomena for the fusion-related research formulated by the Grad-Shafranov equation (GSE) and quantum structures for nanodevices formulated by the Schrödinger equation. GSE has been solved for certain simple excitation types and the corresponding distribution of flux has been obtained. Some illustrative numerical results for the rectangular plasma and infinite potential well have been presented. This review paper pertains to rather simple geometries well-known in relevant publications, whereas future work will be devoted to more complex geometries corresponding to specific realistic scenarios.

\section{APPENDIX: DERIVATION OF SCHRÖDINGER EQUATION}

Electron captured in a small volume can only have discrete energy levels. Discretisation of energy levels is obvious if the considered volume of space is comparable to de Broglie wavelength.

It is possible to derive spatially dependent Schrödinger equation starting from the classical wave Eq. (Helmholtz equation) [18]:

$$
\nabla^{2} \psi+k^{2} \psi=0
$$

where $\psi$ is the wave function and $k$ is the wave number:

$$
k=\frac{2 \pi}{\lambda}
$$

According to de Broglie, particles with energy $E$ and momentum $p$ also have wave properties, i.e. wavelength: 


$$
\lambda=\frac{h}{p}
$$

where $p$ is the momentum of a wave-particle defined as:

$$
p=m v
$$

Now, it simply follows:

$$
k=\frac{2 \pi}{\lambda}=\frac{2 \pi p}{m}=\frac{2 \pi m v}{h}
$$

Inserting (A.5) into (A.1) yields:

$$
\nabla^{2} \psi+\frac{4 \pi^{2} m^{2} v^{2}}{h^{2}} \psi=0
$$

The total energy of a quantum particle can be expressed as the sum of its kinetic and potential energy, respectively:

$$
E=\frac{1}{2} m v^{2}+V
$$

Now combining (A.6) and (A.7), it follows:

$$
-\frac{\hbar^{2}}{2 m} \nabla^{2} \psi+V \psi=E \psi
$$

where:

$$
\hbar=\frac{h}{2 \pi}
$$

Expression (A.8) represents the three-dimensional Schrödinger equation. One-dimensional version is then:

$$
-\frac{\hbar^{2}}{2 m} \frac{\partial^{2} \psi(x)}{\partial x^{2}}+V(x) \psi(x)=E \psi(x)
$$

and, for some scenarios, it can be solved analytically.

\section{REFERENCES}

[1] D. Poljak, Advanced Modeling in Computational Electromagnetic Compatibility, Wiley Interscience, New York 2007. DOI: 10.1002/0470116889

[2] P.P. Silvester and R.L. Ferrari, Finite Elements for Electrical Engineers, 3 $3^{\text {rd }}$ Edition, Cambridge University Press, Cambridge 2001. DOI: 10.1017/CB09781139170611

[3] F. Felici et al., Real-time physics-model-based simulation of the current density profile in Tokamak plasmas, Nuclear Fusion, Vol. 51, 2011.

DOI: $10.1088 / 0029-5515 / 51 / 8 / 083052$

[4] R.R. Khayrutdinov and V.E. Lukash, Studies of plasma equilibrium and transport in a Tokamak fusion Device with the inverse-variable technique, Journal of Computational Physics, Vol. 109, pp. 193-211, 1993. DOI: 10.1006/jcph.1993.1211 
[5] S.H. Aydin and M.Tezer-Sezgin, Numerical Solution of Grad-Shafranov equation for the distribution of magnetic flux in nuclear fusion devices, Turkish J. Eng. Env. Sci., Vol. 32, pp. 265-275, 2008.

[6] R. Zanino, Advanced Finite element modeling of the Tokamak plasma edge, Journal of Computational Physics, Vol. 138, pp. 881-906, 1997. DOI: 10.1006/jcph.1997.5848

[7] E. Fable et al., A stable scheme for computation of coupled transport and equilibrium equations in Tokamaks, Nuclear Fusion, Vol. 53, 2013.

DOI: $\underline{10.1088 / 0029-5515 / 53 / 3 / 033002}$

[8] G. Li and N.R. Aluru, Hybrid techniques for electrostatic analys of nanowires, IEEE 2004. DOI: $\underline{10.1109 / \text { iccad.2004.1382579 }}$

[9] A. Udal, R. Reeder, E. Velmre and P. Harrison, Comparison of methods for solving the Schrödinger equation for multiquantum well heterostructure applications, Proc. Estonian Acad. Sci. Eng., Vol. 12, No. 3-2, pp. 246-261, 2006.

[10] F. Gelbard and K.J. Malloy, Modeling quantum structures with the boundary element method, Journ. Comp. Physics, Vol. 172, pp. 19-39, 2001. DOI: 10.1006/jcph.2001.6751

[11] W. Moy, M.A. Carignano and S. Kais, Finite element method for finite-size scaling in quantum mechanics, J. Phys. Chem. A, 1/2, pp. 5448-5452, 2008. DOI: $10.1021 / \mathrm{jp} 800346 \mathrm{z}$

[12] D. Poljak, A. Rubić and E. Maze, A note on the use of domain discretization methods in modeling of some phenomena in engineering physics, SpliTECH 2017.

[13] D. Poljak and V. Dorić, A Note on FEM Modeling of Some Magnetohydrodynamics Phenomena for Application in Fusion Related Research, 2014 International Conference on Software, Telecommunications and Computer Networks, Split: FESB, University of Split, 2014. DOI: $\underline{10.1109 / \text { softcom.2014.7039063 }}$

[14] S. Sesnić, D. Poljak and E. Slišković, A review of some analytical solutions to the GradShafranov equation, 2014 International Conference on Software, Telecommunications and Computer Networks, Split: FESB, University of Split, 2014.

DOI: $10.1109 /$ softcom.2014.7039066

[15] P. Mc Carthy, Analytical solutions to the Grad-Shafranov equation for tokamak equilibrium with dissimilar source functions, Physics of Plasmas, Vol. 6, No. 9, pp. 35543560, 1999. DOI: $10.1063 / 1.873630$

[16] S. Zheng, A. Wootton and E. Solano, Analytical tokamak equilibrium for shaped plasmas, Physics of Plasmas, Vol. 3, No. 3, pp. 1176-1178, 1996. DOI: $10.1063 / 1.871772$

[17] C. Atanasiu, S. Gunter, K. Lackner and I. Miron, Analytical solutions to the GradShafranov equation, Physics of Plasmas, Vol. 11, No. 7, pp. 3510-3518, 2004.

[18] J. Baggot, The Meaning of Quantum Theory, Oxford University Press, New York, 1992. 